\title{
EL REGRESO DEL FMI AL ECUADOR Y LA RETÓRICA DEL FEMINISMO EMPRESARIAL: LOS POSIBLES EFECTOS EN LA VIDA DE LAS MUJERES
}

\author{
THE IMF'S RETURN TO ECUADOR AND THE RHETORIC OF BUSINESS FEMINISM: \\ THE POSSIBLE EFFECTS ON WOMEN'S LIVES
}

NORA FERNÁNDEZ, ${ }^{1}$ ALEJANDRA SANTILLANA ORTIZ ${ }^{2}$

${ }^{1}$ Pontificia Universidad Católica del Ecuador; ${ }^{2}$ Instituto de Estudios Ecuatorianos

Recepción manuscrito: 30 de septiembre de 2019

Aceptación versión final: 14 de octubre de 2019

\begin{abstract}
RESUMEN Con la llegada de Rafael Correa a la presidencia del Ecuador en 2006, el discurso posneoliberal parecía dejar atrás las políticas de ajuste estructural. Sin embargo, ya en el 2014, el Fondo Monetario Internacional (FMI) regresa a monitorear la economía y en el actual mandato de Lenín Moreno el Estado ecuatoriano firma un nuevo acuerdo. Este artículo busca analizar la Carta de Intención firmada en 2019, desde una crítica marxista y feminista. Se centra en el pacto neoliberal impulsado por el gobierno; analizaremos el rol del FMI en la economía para la región y la retórica multilateral de inclusión de género; y realizaremos una crítica detallada de lo que la nueva carta de intención significa para las mujeres en Ecuador.

palabRas ClaVe Marxismo, economía feminista, instituciones internacionales, equidad de género.
\end{abstract}

ABSTRACT Rafael Correa's ascent to Ecuador's presidency and his post neoliberal discourse showed an opportunity to leave behind the structural adjustment policies. However, by 2014, the IMF had returned to monitor the ecuadorian economy and during the current Lenín Moreno's government an new agreement with IMF has been signed in 2019. This article seeks to analyze the Letter of Intent from a critic marxism and feminist perspective. For this purpose, we (1) analyze the neoliberal covenant promoted by government; (2) discuss the IMF's role in the economy region and the multilateral rhetoric of gender inclusion; (3) finally, we examine the meaning of IMF agreement for the women in Ecuador.

KEYWORDS Marxism, feminist economy, international institutions, gender equity.

JEL CODES B51, B54, F33, J24.

\section{INTRODUCCIÓN}

El retorno del Fondo Monetario Internacional (FMI) a la economía del Ecuador nos plantea el desafío de comprender el nuevo acuerdo firmado entre el Estado ecuatoriano y el organismo 
multilateral. Este artículo busca problematizar la Carta de Intención firmada el 2019 por el Gobierno desde un enfoque crítico que pone en diálogo una perspectiva de economía feminista y una lectura marxista del funcionamiento del capitalismo, el patriarcado y el neoliberalismo.

Se propone una problematización de la Carta de Intención que incluye datos descriptivos sobre el mercado laboral y los recortes presupuestarios en el país así como el caso de Argentina como referencia de los posibles impactos de las nuevas políticas de ajuste en Ecuador. Se incorpora, además, una revisión de declaraciones y documentos que muestran el discurso del FMI en relación a las mujeres. Interesa preguntarse ¿cuáles son los elementos de la Carta de Intención que revelan los propósitos del FMI para las mujeres en el país?, ¿cómo este acuerdo se relaciona con la retórica del FMI en relación a las mujeres y el emprendedurismo? y, finalmente, ¿qué posibles consecuencias tiene este acuerdo en la vida de las mujeres del Ecuador?

La hipótesis de este estudio es que al ser el neoliberalismo un proyecto de clase de las élites globales, que se articula con las élites nacionales y solo es posible con el accionar de los Estados; requiere no solo de políticas de ajuste estructural dirigidas a la clase trabajadora, sino también de la legitimación de un discurso de aparente inclusión con perspectiva de género, pero basado en un feminismo neoliberal que borra las condiciones de desigualdad e injusticia, y la división sexual del trabajo que estructuran el capitalismo y se alimentan del patriarcado como orden social, pero también económico, que plantea que las mujeres pueden liberarse de la pobreza y de la violencia, únicamente con voluntad y cumpliendo con el rol del Homo economicus del capital. De esta manera, las mujeres son afectadas tanto por la deuda que contrae el Estado, y que recae directamente en el recorte de presupuestos de inversión social, como por la deuda privada, que se convierte en el mecanismo de sobrevivencia ante los efectos de las políticas de ajuste.

\section{EL PACTO ENTRE LAS ÉLITES INTERNACIONALES: NEOLIBERALISMO Y FONDO MONETARIO INTERNACIONAL}

Tu mujer emprendedora, es una ajustadora. PINTADO EN LAS PAREDES DE BUENOS AIRES (NOVIEMBRE 2018)

Desde los años setenta del siglo pasado, en que el neoliberalismo irrumpió en América Latina a través de su primer experimento en el régimen militar de Pinochet y cobró vigencia a nivel global como una teoría de prácticas político-económicas, éste se ha encaminado a «restablecer - o crear - las condiciones para la acumulación del capital y restaurar el poder de las élites económicas», reiteradamente amenazadas por la caída de las tasas de ganancia y las subsecuentes crisis inherentes del capitalismo (Harvey, 2014, pág. 36).

Si bien es cierto, las crisis económicas y financieras — cada vez más recurrentes_- ponen en duda la capacidad del neoliberalismo como proyecto político para vigorizar el proceso global de valorización y acumulación del capital, no cabe duda su capacidad para renovar el poder de las élites económicas (Harvey, 2014). Basta ver las cifras de Oxfam (Lawson, Chan, Rhodes, Parvez Butt, Marriott, Ehmke, Jacobs, Seghers, Atienza y Gowland, 2019) sobre el aumento de 
la desigualdad extrema, que muestran cómo desde el inicio de la crisis económica-financiera del 2008, el número de multimillonarios prácticamente se ha duplicado.

Esta restauración del poder económico de las clases altas no hubiera sido posible sin la captura del poder político realizado por las élites mundiales a través del apresamiento de los Estados y las instituciones del sistema financiero internacional, fundamentalmente el FMI y el Banco Mundial (BM) convertidos desde la era Reagan en «centros para la propagación y la ejecución del fundamentalismo del libre mercado y la ortodoxia neoliberal» (Harvey, 2014, pág. 46).

Recesiones inducidas, recortes de gasto social, creación de legislaciones que flexibilizaron el mercado laboral y debilitaron el poder de los sindicatos, privatizaciones y rebajas de los impuestos a las empresas y élites económicas, convirtieron a las políticas de ajuste estructural impulsadas por los Estados en condiciones para acceder a los programas de reprogramación de la deuda, consolidando el poder de los monopolios y el capital financiero global, así como el rechazo de la población afectada por la pobreza, desposesión y desigualdad generada a partir de dichas medidas (Harvey, 2014).

\section{EL NUEVO ROSTRO DEL FMI: INCLUSIÓN, DISCURSO DE GÉNERO Y FEMINISMO EMPRENDEDOR NEOLIBERAL}

Luego de décadas de desprestigio global y después de la crisis del 2008, el FMI cambió su discurso por uno más enfocado al combate de la pobreza y la desigualdad, e incorporó «la inclusión de las mujeres» (Lagarde, 2016). En sus intentos por convencer a la población de que sus rescates económicos tienen un profundo contenido social, que busca beneficiar a los sectores mayoritarios, su exdirectora, Christine Lagarde, declaró públicamente que esta entidad tenía un nuevo rostro y que ya no imponía ajustes estructurales a los países con dificultades económicas (Emol.com, 2014; El País, 2018).

Mientras presentaba su nuevo discurso al mundo, el FMI continuaba firmando cartas de intención y memorándums de políticas económicas y financieras cuyo trasfondo seguía siendo el ajuste estructural como única alternativa para enfrentar las crisis en los países europeos. La misma receta: privatizaciones, despidos, recortes de pensiones, flexibilización laboral y financiera. Las mismas consecuencias: recesión económica, precarización laboral y empobrecimiento de la mayoría de la población. En otras palabras, una nueva máscara, las mismas políticas, el mismo FMI, la misma alma.

¿A qué alma se hace referencia? A la señalada por Margaret Thatcher en su famosa frase «la economía es el método, la finalidad es cambiar el corazón y el alma» (Thatcher, 1981). Pero ¿dónde se puede ver el alma del neoliberalismo? En el marco teórico-conceptual a partir del cual opera el FMI, que sostiene que si los agentes económicos, empresas y consumidores, maximizan sus beneficios y utilidades de manera individual, a la vez que el Estado garantiza la propiedad privada y deja que las fuerzas del mercado operen libremente, la sociedad alcanzará su máximo bienestar (FMI, 2019). En el alma del Homo economicus que representa al emprendedor moderno, racional, egoísta, calculador, competitivo, individualista, cuyo objetivo es siempre satisfacer sus deseos y en cuyo mundo, tal como lo señalará Thatcher, ${ }^{1}$ no hay sociedad, solo individuos (Marcal, 2018, págs. 29-49; Himmelweit, 2018, pág. 63). 
El Homo economicus es un hombre abstracto, sin raza, clase y en donde lo masculino se presenta como universal, cuyas características son lo más alejadas posibles a lo que representa lo femenino como construcción social: emocional, solidaria, abnegada y dispuesta a cuidar a los demás renunciando a maximizar su propio beneficio, por ende, poco competitiva y productiva, razón por la cual es excluida del mercado y su trabajo debe recibir ninguna o menor remuneración (Marcal, 2018, págs. 29-49). Este marco conceptual garantiza que el poder económico siga siendo masculino y sus empresas continúen acumulando el capital. El mismo marco teórico que, en palabras de Rita Segato (2019), «recrudece las prácticas patriarcales» mientras beneficia fundamentalmente a las grandes corporaciones y el capital financiero internacional». Tres días antes de que tenga lugar el Foro Económico Mundial en 2018, Christine Lagarde, exdirectora del FMI, y Elsa Solberg, primera ministra de Noruega, escribían un artículo titulado «¿Por qué 2018 debe ser el año en que las mujeres prosperen?», para ellas:

Este año, en el Foro Económico Mundial, el desafío del empoderamiento femenino también está firmemente en la agenda, porque darles a las mujeres y las niñas la oportunidad de tener éxito no solo es lo correcto, sino que también puede transformar las sociedades y la economía. (Lagarde y Solberg, 2018)

Desde hace un par de años, la presencia de mujeres en los foros y grupos donde convergen las naciones y empresas más poderosas se vuelve cada vez más visible. Tanto, que la mayoría de instituciones multilaterales y de articulaciones que marcan la agenda de las políticas mundiales han incluido a las mujeres. ¿Por qué de pronto importamos estos organismos y espacios? En nuestro criterio, tres son los elementos que explicarían este rostro femenino del capital internacional: dos de tipo político-ideológico y un tercero que se rige por las reglas del orden económico del modo de producción capitalista. Los tres factores se encuentran en tensión y ocurren de manera simultánea mostrando que el capitalismo es un orden social en donde el patriarcado y la colonialidad establecen a la par, divisiones y jerarquías, también entre mujeres.

Se considera que los elementos de carácter político e ideológico que expresan esta centralidad repentina de las mujeres en el discurso y las figuras internacionales de la gobernanza, son: (1) la consolidación de los argumentos y el surgimiento de referentes de un tipo de feminismo neoliberal que defiende la igualdad relativa entre hombres y mujeres - mientras evita hablar de la incompatibilidad con la desigualdad e injusticia generada por el capitalismo-; y (2) la neutralización del potente y plural movimiento feminista que en la actualidad constituye el movimiento con mayor capacidad de movilización simultánea e internacionalista a nivel global, porque propone en la movilización, una crítica profunda y sistémica a las relaciones sociales de producción, la división sexual del trabajo, la racialización y despojo, a la vez que hace frente al fascismo y la derechización en el mundo.

En cuanto al tercer factor, directamente relacionado con lo económico, la inclusión de género y la incorporación de las mujeres al mercado de trabajo productivo bajo las nuevas formas de contratación, promovida por los organismos multilaterales, recogen la histórica herramienta del capitalismo de reducir costos de producción aprovechando el lugar subordinado y de sobrecarga de trabajo de cuidado de las mujeres en las sociedades (Martínez, 2011). Como 
bien lo han propuesto las feministas marxistas en sus investigaciones (Arruzza, 2016; Federici, 2010; Davis, 2004), la relación entre el capitalismo, el patriarcado y la racialización ha implicado que el rol de las mujeres en la economía esté determinado por su lugar en la división sexual del trabajo. Este mecanismo usado por el capitalismo, para aumentar las ganancias de los empresarios, se basa en el carácter potencial de las mujeres para ser madres y, por lo tanto, en la posibilidad futura de que queden temporalmente fuera del mercado laboral productivo. Cuando esto ocurre, no pueden proveer de fuerza de trabajo a las empresas - aunque sí mantienen su trabajo en la reproducción social reforzado con la maternidad-, y son el Estado y las empresas quienes están llamados a asumir el costo de la fuerza de trabajo productiva temporalmente no disponible.

Bajo ese argumento, las mujeres que tienen la carga de trabajo no remunerado, ocupan un lugar en el mundo del trabajo asalariado potencialmente más costoso que los hombres y es bajo esta premisa que se refuerza su condición subordinada en el orden capitalista. Esto no es menor cuando de políticas neoliberales se trata.

Como se verá más adelante, la entrada al mercado laboral asalariado neoliberal de las mujeres y los jóvenes está condicionada a una forma de contratación que legitima y formaliza la flexibilización laboral, con menos remuneración y con mayores posibilidades de ser despedidos. Y es que, en el capitalismo, ningún empresario quiere asumir los costos de garantizar los derechos de maternidad; pero en regímenes neoliberales, el Estado tampoco, porque traslada obligatoriamente la gestión de la reproducción social y de la maternidad a las mujeres y sus entornos (Schild, 2016), ocurre aquello que la economía feminista ha denominado crisis de los cuidados, es decir, se produce una tensión en la provisión de cuidados derivada de los cambios en el rol de las mujeres en la economía, de la organización capitalista y patriarcal del trabajo de cuidado y del retiro del Estado en las esferas sociales (Hochschild, 1995). En efecto, esta crisis se relaciona con «los cambios en los contenidos, protagonistas y circunstancias en las que ese trabajo se realiza » (Hochschild, 1995, págs. 331-346) que en contextos neoliberales implica, mercantilización.

Los elementos ideológicos, políticos y económicos estructurales que aquí se muestran, no surgen espontáneamente. Son respuestas y estrategias del capitalismo y el patriarcado para aumentar ganancias y mantener el orden material y simbólico. Asimismo, constituyen mecanismos para contrarrestar cualquier posibilidad de concretar horizontes emancipatorios en el plano de la organización. En este caso, frenar el crecimiento y la radicalización del movimiento internacionalista feminista anticapitalista y anticolonial. En estos años, las élites mundiales han creado un discurso sobre la importancia de la participación de las mujeres en puestos de dirección política y gerencial a la par de una promesa emprendedora para las mujeres trabajadoras. El discurso empresarial sustentado en varios estudios de aumento de rentabilidad, apela a que nosotras somos más responsables, mejores gerentes y contratamos a más mujeres (Revista Líderes, 2016; Revista Líderes, 2018).

Es así que en 2017 el Foro Económico Mundial (WEF) en alianza con LinkedIn, incorporó en el Global Gender Gap Report un análisis sobre la participación de las mujeres en puestos de liderazgo en 12 industrias con el propósito de comprender las tendencias de género en la composición de la fuerza de trabajo. Concluyó que las mujeres representan no más del 50\% 
de los líderes en todas las industrias analizadas, y en algunas áreas como energía, mina o manufacturas, la participación de las mujeres en cargos de representación es mucho más baja, y solo constituye el $20 \%$ de los puestos de liderazgo (LinkedIn, 2017).

El estudio demuestra que en el período 2007-2017, la participación femenina en cargos altos ha crecido aproximadamente a un ritmo del $2 \%$, y recomienda que para acelerar esta tendencia es fundamental colocar a mujeres en cargos de liderazgo porque éstas contratan a más mujeres. La investigación también determina que solo en tres industrias las mujeres asumen el 40\% de los cargos de liderazgo corporativo: salud, educación y sectores que no generan ganancias. Sin embargo, estas industrias han sido históricamente feminizadas por el número relativo de mujeres trabajadoras en esos sectores y porque tienen monetaria y socialmente una menor paga y un menor reconocimiento (WEF, 2017). Pero ¿cuál es la razón que está detrás de esta promoción de las mujeres en puestos de liderazgo?

Recuérdese que más allá de la presencia de pocas mujeres vinculadas o representantes directas de las empresas transnacionales en puestos de dirección o de una mayor participación de estas en los distintos sectores de la economía, la gran mayoría de las mujeres y las identidades sexo genéricas feminizadas, son fuerza de trabajo explotada y empobrecida (Oxfam, 2017). Sin embargo, la narrativa del feminismo emprendedor neoliberal cuenta en los últimos tres años con una serie de publicaciones ${ }^{2}$ promovidas y financiadas por grupos económicos mundiales que recopilan frases y experiencias de las mujeres más poderosas que son parte de la lista Fortune $500^{3}$ (Ortega, 2018). El argumento que subyace en esta campaña mediática es que tanto el patriarcado como la pobreza son superables con voluntad individual y empresarial, es decir, las mujeres pueden ser exitosas en el mercado si adoptan las características del Homo economicus.

Bajo esa lógica, para superar las brechas de género, se debe incluir a más mujeres al ámbito político, gerencial, laboral y de régimen de propiedad. En efecto, las salidas que proponen los organismos multilaterales se concentran en tres mecanismos: mayor participación de mujeres en la fuerza de trabajo para impulsar el PIB, más mujeres en puestos de dirección y defensa de los derechos de propiedad ${ }^{4}$ (Lagarde y Ostry, 2018). Estos planteamientos también los encontramos en lo establecido por el Grupo de los 20 (G2O), que emite un documento denominado Women 20, para el tratamiento de las mujeres en los acuerdos de las grandes potencias mundiales.

Como señala la Revista Líderes (2018), edición mexicana, una mayor participación de las mujeres en puestos de liderazgo no solo tiene que ver con el acceso a la cultura y educación, sino fundamentalmente está relacionado con un aumento de la rentabilidad de las empresas. Mientras menos participen mujeres en puestos de liderazgo, se limita el crecimiento de la economía nacional y, por lo tanto, de ganancias y oportunidades para las empresas. Las mujeres reciben mayor presión social para demostrar que son más capaces, que sumada a la brecha salarial de género determinan «una ganancia por empleado 30\% mayor y una rentabilidad más alta que sus competidores» (Líderes, 2018).

Otro estudio realizado por el Peterson Institute of Economy (como se citó en Líderes, 2018), basado en «21.980 encuestas realizadas a empresas globales que cotizan en la bolsa en 91 países de diversas industrias, concluye que tener al menos un 30\% de mujeres en puestos de alta dirección en una organización añade $6 \%$ más de margen de utilidades netas». 
Este discurso de democratización e inclusión de género que promueve el capital financiero y comercial internacional, que se muestra sensible a las demandas levantadas por gran parte de los movimientos feministas desde los años sesenta, muestra la enorme capacidad para vaciar de contenido progresista las exigencias de la clase trabajadora y rentabilizarlas. Es por eso, que el carácter radical del movimiento feminista actual se encuentra justamente en que ya no esgrime únicamente la demanda de igualdad, sino de transformación de todo el sistema ${ }^{5}$ (Development Alternatives with Women for a New Era (DAWN), 2018).

Como se ha visto el contenido de este feminismo neoliberal del emprendedurismo, promovido y encarnado por las mujeres del capital financiero y comercial mundial, reconoce la importancia de la participación femenina, pero se sostiene en la desvalorización estructural de las mujeres y de la naturaleza, del proceso de reproducción y del sostenimiento y cuidado de la vida - y de todo aquello que la posibilita-, así como de las bases de explotación y dominación en la que se erige el capitalismo.

\section{EL FMI DURANTE EL GOBIERNO DE RAFAEL CORREA: CONTINUIDADES DEL CARÁCTER DEPENDIENTE DE LA ECONOMÍA PERIFÉRICA ECUATORIANA}

Desde el inicio de su mandato, el entonces presidente del Ecuador, economista Rafael Correa Delgado, sostuvo que uno de los objetivos centrales de la Revolución Ciudadana era la superación de «la larga noche neoliberal» (CELA, 2007; La Jornada, 2009), que implicaba colocar al ser humano en el centro de las políticas públicas, y no al capital — sobre todo financiero- y su lógica depredadora. Ese fue el sentido que se recogió en la Asamblea Nacional Constituyente de 2007 y que sirvió de texto constitucional, aprobado en 2008.

De igual manera, el primer Plan Nacional del Buen Vivir incluyó la importancia de la justicia social, de la construcción igualitaria y de la promoción y garantía de derechos que aporten en la superación de las desigualdades y discriminaciones de clase, raza o género (2009). Cuatro años después, el expresidente Correa declaraba en una conferencia en la Universidad Técnica de Berlín, que «el FMI es el perro guardián del capital financiero» (Correa, 2013, pág. 29). Sin embargo, como lo mostró el boletín del 28 de mayo de 2014 emitido por el mismo Fondo Monetario Internacional, éste retomaba su papel en la revisión de la economía ecuatoriana, que en concreto significaba el «cumplimiento del mandato establecido en el Artículo IV del Convenio Constitutivo del FMI» ${ }^{6}$ (Acosta, 2014).

Este retorno, que no significó un acuerdo entre el Gobierno del Ecuador y el FMI, constituye, sin embargo, un ejemplo claro del carácter dependiente de la economía nacional y del poco margen de acción y decisión de quienes administran el Estado frente a la hegemonía del neoliberalismo como forma concreta del capitalismo global. Solo con el monitoreo del FMI a nuestra economía, y la elaboración de un informe favorable, Ecuador estaba habilitado para emitir los Bonos $2024^{7}$ y obtener recursos del mercado de capitales global para hacer frente al déficit fiscal consecuencia de seguir siendo una economía primario-exportadora-dependiente y como producto de la caída de los precios internacionales del petróleo.

Entre las medidas para enfrentar la recesión, el Gobierno realizó una serie de reformas regresivas en términos de derechos laborales tanto para el sector público como privado, ${ }^{8}$ es así 
que se alineó a las necesidades del neoliberalismo hegemónico global que se caracteriza por «consolidar una sociedad bilateral entre el capital y el trabajador, dinamitando el esquema de negociación colectiva, dejando vacío de contenido al sindicalismo y situando el concepto de movimiento obrero en las vitrinas de los museos» (Cañamero, 2018, pág. 7).

\section{EL RELATO DE LA CRISIS: ENTRE EL «NO HAY ALTERNATIVA» Y LA VIOLACIÓN CONSTITUCIONAL DE DERECHOS}

En julio de 2017, mientras las cifras oficiales del Banco Central del Ecuador, mostraban que el Ecuador se encontraba en etapa de cierta recuperación económica (BCE, 2017); el presidente Lenín Moreno anunciaba que el país atravesaba por una difícil situación económica, como consecuencia de la falta de mesura en el gasto público de la administración correísta. Ante esto, se volvía necesaria la «optimización de la inversión pública, incentivar la inversión y tomar un conjunto de medidas de austeridad fiscal» (El Universo, 2017). Con este discurso, se inaugura la estrategia del Gobierno para construir consenso y legitimidad social en torno a sus medidas económicas, apoyándose para ello en la profecía autocumplida de que, frente a la crisis, «no hay alternativa ${ }^{9}$ posible a las políticas neoliberales.

En junio de 2018, el Gobierno contó con la aprobación por parte de la Asamblea Nacional de la Ley Orgánica para el Fomento Productivo, Atracción de Inversiones, Generación de Empleo y Estabilidad y Equilibrio Fiscal. A través de la remisión tributaria, esta ley benefició a los grandes deudores del fisco y puso las bases para un futuro acuerdo con el FMI, entidad que mostró su satisfacción con las medidas económicas a través de las declaraciones de la jefa de la misión en 2018: «Las autoridades ecuatorianas han dado, recientemente, pasos importantes para fortalecer las instituciones fiscales y restablecer una economía competitiva impulsada por el sector privado» (FMI, 2018).

En consonancia con el discurso de la inevitabilidad del retorno neoliberal, encaminado a mostrar al FMI el compromiso del país con el ajuste, se tomaron acciones previas ${ }^{10}$ a la firma del acuerdo, como el aumento del precio y la liberalización del mercado de la gasolina súper, el despido de miles de empleados públicos, ${ }^{11}$ la restricción de la inversión pública en capital y el recorte del presupuesto público para el año 2019.

Finalmente, en febrero de 2019, el Gobierno anuncia que el Estado ecuatoriano y el FMI ha logrado un acuerdo técnico. Con esto, el país recibía un préstamo de USD 4200 millones $^{2}$ para ejecutar las políticas económicas del Gobierno en el marco del servicio ampliado ${ }^{13}$ del FMI (SAF) (FMI, 2019b). Según este marco, se espera que los compromisos del país estén focalizados en el ajuste estructural e incluyan la condicionalidad específica ${ }^{14}$ (FMI, 2016).

Cabe decir que el regreso del FMI abre la puerta a mayor endeudamiento del Estado ecuatoriano y más dependencia al sistema financiero global, es decir, obliga a que nuestro trabajo social sirva para pagar a tenedores de deuda y organismos multilaterales, y no para generar riqueza que se distribuya a la población.

Este acuerdo se logra violando la Constitución vigente que en su artículo 419 determina que cualquier tipo de acuerdo internacional debe contar con la aprobación previa de la Asamblea Nacional cuando éste requiera modificar una ley, se refiera a derechos y garantías establecidas 
en la Constitución, se «comprometa la política económica del Estado establecida en el Plan Nacional de Desarrollo a condiciones de instituciones financieras internacionales o empresas transnacionales» y que «atribuyan competencias propias del orden jurídico interno a un organismo internacional o supranacional» (Constitución, 2008, pág. 185).

La Carta de Intención con el FMI firmada por Moreno, así como el Tratado de Libre Comercio con la Unión Europea ${ }^{15}$ suscritos durante el Gobierno de Correa, constituyen acuerdos que vulneran los principios de solidaridad y justicia, así como varios derechos establecidos en la Constitución. ${ }^{16}$ Es decir, en ambos gobiernos se violan derechos constitucionales en pro del pacto con las élites económicas.

\section{LA NUEVA CARTA DE INTENCIÓN CON EL FMI: UNA LECTURA DESDE EL FEMINISMO CRÍTICO Y EL MARXISMO}

El capital financiero internacional junto a su discurso de inclusión de género, se traduce en el caso ecuatoriano en los contenidos de la nueva Carta de Intención firmada entre el Gobierno de Moreno y el FMI. El Memorando de Políticas Económicas y Financieras, que resume el Plan Prosperidad, se divide en seis secciones que justifican su alcance a partir de las bondades de la competitividad, la reducción del Estado y la incorporación de las mujeres y jóvenes al mercado laboral asalariado.

A continuación se realizará una lectura de los posibles efectos en las mujeres de las políticas contenidas en las distintas secciones del acuerdo: a) Restaurar la prudencia en la política fiscal; d) Apoyo a la creación de empleo, la competitividad y el crecimiento; y e) Promover la prosperidad compartida y proteger a pobres y vulnerables.

\section{PRECARIZADAS Y SOBREEXPLOTADAS}

La reforma laboral pactada entre el FMI y el Gobierno del Ecuador se compone de tres elementos centrales: (1) flexibilización laboral sobre todo enfocada a mujeres y jóvenes; (2) reforzamiento de las mujeres en el trabajo reproductivo no remunerado en condiciones de mayor precarización y sobreexplotación; y, (3) más despidos y reducción de los salarios en el sector público.

En cuanto al primer elemento, como establece la sección D de la Carta de Intención, el Gobierno busca devolverle competitividad internacional a la economía ecuatoriana basada en fomentar un crecimiento liderado por el sector privado. El propósito final es hacer del Ecuador «un destino preferido para los negocios de todo el mundo e impulsar la generación de empleo y la reducción de la informalidad en la economía» (LOI-MEFP, 2019, pág. 9). Para lograrlo, necesita modificar el marco laboral en el que se encuentra el país:

Ecuador requiere una reforma laboral urgente que ayude a reducir la informalidad — particularmente de mujeres y de trabajadores jóvenes- e incremente la predisposición de los emprendedores y las empresas para contratar trabajadores y crecer. Una reforma laboral implica adaptarse a las condiciones del mercado y de la sociedad retirando cuidadosamente las restricciones actuales que resultan en menores oportunidades para las personas que no tienen un empleo [...]. (LOI-MEFP, 2019, pág. 9) 
Esta propuesta omite las condiciones de contexto por las mujeres ecuatorianas enfrentan la informalidad. De acuerdo a datos del Instituto Nacional de Estadísticas y Censos (INEC, 2019), el trabajo no remunerado (TNR) está fundamentalmente en manos de las mujeres. Es así que, en el período 2007-2017, por cada 100 horas de TNR, ${ }^{17}$ las mujeres realizaron 77. Para el año 2017, mientras que el TNR contribuyó con el 32\% del gasto del consumo final de los hogares a nivel nacional ${ }^{18}$ y el $19,1 \%$ del $\operatorname{PIB}^{19}-14,5 \%$ generado por las mujeres y $4,6 \%$ por los hombres-, las mujeres de 12 años en adelante dedicaron en promedio 26,7 horas per cápita por semana al TNR, a la vez que los hombres solo le asignaron 8,2 horas. Esto significa que las mujeres trabajan sin remuneración tres veces más que los hombres en aquellas actividades que facilitan la reproducción y sostenimiento de la vida.

Si se suma las actividades del TNR y el trabajo remunerado de las personas ocupadas en el mercado, la carga global de trabajo de las mujeres es mayor a la de los hombres en 17,5 horas por semana, lo cual significa menos tiempo para actividades «que las beneficien en términos individuales — recreación, educación, descanso-» (Consejo Nacional por la Igualdad de Género (CNIG), 2016, pág. 30). ${ }^{20}$ Según Castillo y Salas (2018):

La desigualdad en la distribución del tiempo en actividades domésticas y cuidado de cargas familiares (principalmente los niños pequeños), disminuye la oferta laboral de mujeres, generando condiciones inequitativas en el desarrollo profesional, aún en aquellos sectores con mayor participación femenina. Las mujeres con baja cualificación son menos propensas a acceder a un trabajo, y entre aquellas que trabajan, la intensidad laboral y los salarios son inferiores que sus pares hombres [...]. (págs. 174-17)

En ese contexto de enormes desigualdades de género, retirar las restricciones actuales en el mercado laboral, que en lenguaje neoliberal significa una mayor flexibilización laboral para mujeres sin impulsar cambios en las condiciones estructurales que configuran dicha desigualdad, termina constituyéndose en una política que legitima su mayor explotación y precarización.

Un poco más adelante, en la misma sección D de la Carta, que presenta la promoción de la igualdad de género como parte de las reformas necesarias, se señala:

Continuaremos con nuestras políticas de apoyo a las familias con niños pequeños, asegurando la provisión de programas de cuidado de niños. La introducción de contratos laborales menos rígidos beneficiará en particular a las mujeres que prefieran trabajar a tiempo parcial o en empleos temporales, lo cual aumentará su participación en el mercado laboral. Otras políticas encaminadas a lograr un mercado laboral más dinámico ayudarán a estimular el crecimiento del sector servicios, lo cual apoyará la participación de la mujer en el mercado laboral. (LOI-MEFP, 2019, pág. 10) (Las cursivas son nuestras)

Este acuerdo afirma que generando contratos más flexibles se favorecerá a las mujeres, quienes tendrían mayores posibilidades de insertarse en el mercado laboral, combinando su trabajo de cuidado no remunerado con el trabajo remunerado. Este anclaje de la flexibilización laboral, los multiempleos y la reducción de horas laborales remuneradas con la maternidad, produce un reforzamiento del rol de las mujeres en la división sexual del trabajo que, al no tomar en cuenta el contexto actual de recesión de la economía ecuatoriana, ${ }^{21}$ desconoce que 
son precisamente las mujeres embarazadas, las madres, así como las más jóvenes y las disidencias, las más vulnerables.

Aunque en su estudio, Castillo y Salas (2018) concluyen que en Ecuador «los patrones de fluctuación de empleo por género en relación a la expansión y contracción económica son relativamente similares y no se observan diferencias en términos de vulnerabilidad del empleo según el ciclo» (pág. 174), los datos de la Encuesta Nacional de Empleo, Desempleo y Subempleo (Enemdu) de diciembre de 2019, muestra que aunque para ambos géneros hay una caída en las tasas de empleo adecuado, la brecha a favor de los hombres entre 2018 y $2019^{22}$ aumenta de $14 \%$ a $14,2 \%$; mientras que para ambos sexos la tasa de empleo no remunerado aumenta, ${ }^{23}$ la brecha a favor de las mujeres en ese mismo período aumenta de $11,7 \%$ a $12,9 \%$. Es decir, se mantiene la tendencia estructural de que los hombres acceden más a empleos en condiciones plenas y las mujeres a empleos sin remuneración, siendo esta última la cual disminuyó de manera estadísticamente significativa (INEC, 2020). Asimismo, en ambos años, las mujeres acceden más a otros empleos no plenos con una diferencia «a su favor» de $2,7 \%$ en el 2018 y $2,8 \%$ en el 2019, mientras que los hombres se subemplean en tasas mayores pasando de $17,2 \%$ en 2018 a 19\% en 2019, superando al subempleo de las mujeres con $1,7 \%$ y 2,8\%, respectivamente. ${ }^{24}$

En 2019, el nivel de desempleo alcanzó al 3,9\% de la PEA a nivel nacional -la mayor tasa registrada en la última década - afectando por género al 4,6\% de las mujeres y al 3,3\% de los hombres. Por otra parte, mientras que el ingreso promedio de los hombres con empleo es de USD 345,50, el de las mujeres es de USD 292,7; es decir, la brecha salarial por género es de USD 52,80 , siendo menor (2,59\%) a la registrada en el 2018 (USD 63,9). Sin embargo, como señala Baéz (2019), esta aparente mejora en la desigualdad por ingresos «se debe a que existe una disminución del promedio del ingreso laboral en los hombres del 3,4\% en comparación al año 2018 y una leve disminución en el caso de las mujeres del o,3\%».

Con respecto a la calidad del empleo: entre diciembre de 2018 y 2019, el número de empleados con algún tipo de seguridad social disminuyó del 41,8\% al 39,9\%; la participación en el sector formal cayó de $46,2 \%$ al $44,7 \%$, mientras que se registraron aumentos menos significativos en el sector informal (o,5\%), el empleo doméstico (o,1\%) y empleos no clasificados por sector $(\mathrm{o}, 8 \%)$. Este deterioro de los indicadores laborales junto a la mayor carga de trabajo global, genera además una crisis afectiva y de salud en las mujeres, fundamentalmente de sectores populares ${ }^{25}$ que verán intensificada la jornada laboral, y con ella el agotamiento mental y emocional.

En cuanto a los despidos, es fundamental observar que el acuerdo propone bajar sus costos en el sector privado en perjuicio de las y los trabajadores.

[...] Con esta reforma disminuiremos gradualmente los costos implícitos de contratación y generaremos incentivos para la creación de empleos y para el crecimiento de pequeñas y medianas empresas. Esta reforma permitirá que el actual mercado del trabajo converja hacia uno que se adapte adecuadamente a las necesidades de distintos sectores y responda a las necesidades de las mujeres y los jóvenes. (LOI-MEFP, 2019, pág. 10)

Esta reforma laboral busca alinear al Ecuador al neoliberalismo global y su esquema de explotación del siglo xxI, caracterizado por «el desmontaje de la legislación garantista, fraccionamiento del empleo y el salario disponible, y consolidación de una reserva de desempleados 
dispuestos a ser explotados» (Cañamedro, 2018, pág. 7). Tal como señala Zafra (2017) «en el carácter precario de los trabajos disponibles radica la situación ventajosa de quien contrata hoy movido por la maximización racionalista de menor inversión y mayor beneficio» (pág. 15). Adicionalmente, la reducción del gasto corriente propuesta en la sección A del acuerdo, significa más despidos de médicos, enfermeros y maestros y una expropiación de la infraestructura pública, que garantiza condiciones mínimas para las economías de cuidado sostenidas fundamentalmente por mujeres e identidades feminizadas. Si el Estado deja de garantizar derechos, servicios y bienes públicos, es decir, si exterioriza todo aquello que no genera ganancias para los empresarios, son las mujeres quienes deberán asumir las tareas de cuidado y reproducción de la vida frente a la actualización de las tarifas por servicios de las entidades que, como se señala en la Carta de Intención «reflejan con mayor exactitud los costos y los mercados a los que atienden» (LOI-MEFP, 2019, pág. 5), así como ante la privatización de los servicios públicos que se encuentra implícito al fijarse como objetivo «monetizar activos que permanecen bajo propiedad pública» (LOI-MEFP, 2019, pág. 5).

Para la economía feminista, «la reproducción implica una puesta en común de los medios materiales y supone el mecanismo primordial por el cual se crea el interés colectivo y los lazos de apoyo mutuo» (Federici, 2013, pág. 253). En contextos neoliberales, con acuerdos como el firmado con el FMI, las diferencias son usadas como formas de división y jerarquías de género, económicas, étnicas y generacionales, que modifican las condiciones para que pueda sostenerse lo común.

En ese sentido, la reducción de presupuestos para los servicios sociales y la privatización de la infraestructura social implican un incremento del TNR realizado por las mujeres, quienes absorben los recortes en política pública para salud y educación, al «dejar sus ocupaciones para cuidar de los niños y las personas dependientes, pues no hay servicios públicos que lo hagan» (Lawson et al., 2020, págs. 48-49). Este proceso termina siendo un mecanismo de transferencia desde las economías domésticas hacia la acumulación de capital. Como sostiene Corina Rodríguez (2015), es el trabajo reproductivo subsidiando las tasas de ganancias de los capitalistas. Así, las empresas aumentarán sus ganancias debido a la reducción de costos, y el Estado abandonará su responsabilidad en garantizar la vida digna de las familias, delegando el cuidado al ámbito privado y estableciendo una pedagogía de la crueldad y la desesperanza en las mujeres e identidades feminizadas; que para cuidar y permitir la reproducción social, se ven obligadas a percibir menos reconocimiento monetario o mayor precarización laboral.

Por su parte, la reforma fiscal - la ampliación del número de contribuyentes y el aumento de impuestos indirectos como el IVA- a la que se ha comprometido el Gobierno, contribuirá al detrimento de las condiciones de vida de las clases medias y populares, mientras que los más ricos verán reducir sus obligaciones tributarias y podrán sacar la riqueza del país a paraísos fiscales. Esto tendrá efectos sobre la forma de la organización social del cuidado, la misma que está vinculada como experiencia socioeconómica estratificada, resultando «en sí misma un vector de reproducción y profundización de la desigualdad» entre las mujeres (Rodríguez, 2015, pág. 42).

Dentro de la sección E del Acuerdo, donde se detallan las políticas para «Promover la prosperidad compartida y proteger a pobres y vulnerables», el Gobierno se compromete a pasar 
de un sistema social con visión de universalización a uno de asistencia social focalizada, característico de la intervención neoliberal en el Estado, reforzando así «una jerarquía de merecimientos en relación a la obligación de las mujeres según sus roles en la familia patriarcal: tener hijos, cuidarlos, escolarizarlos, vacunarlos» (Gago y Cavallero, 2019). De esta manera, se refuerza nuevamente una crisis de cuidados, que bajo esta perspectiva se refiere al:

[C]omplejo proceso de desestabilización de un modelo previo de reparto de responsabilidades sobre los cuidados y la sostenibilidad de la vida, que conlleva una redistribución de las mismas y una reorganización de los trabajos de cuidados, proceso que está cerrándose actualmente de forma no sólo insuficiente y precarizadora, sino reaccionaria, en la medida en que se basa en los mismos ejes de desigualdad social e invisibilidad de trabajos y agentes sociales que presentaba el modelo de partida. (Pérez Orozco, 2006, págs. 9-10)

Si los cuidados son toda la energía y el trabajo que se destina a «la gestión y el mantenimiento cotidiano de la vida y de la salud, — se podría agregar la alimentación_ la necesidad más básica y diaria que permite la sostenibilidad de la vida» (Pérez Orozco, 2006, págs. 9-10), la precarización que acentúa el neoliberalismo a través de recortes, reducción y flexibilización en el ámbito del trabajo, condiciona la posibilidad de realizar tareas concretas que resuelven, sostienen y gestionan las necesidades materiales y afectivas de los seres humanos. Trabajo que como hemos visto, se encuentra fundamentalmente en manos de las mujeres.

\section{ENDEUDADAS}

El gasto del consumo final de los hogares presentó un crecimiento interanual del 1,1\% durante el tercer semestre del 2019. Entre los factores que explican este resultado están «el desempeño positivo del número de operaciones de crédito de consumo (17,1\%); y, el aumento de $10,1 \%$ de las remesas recibidas, que alcanzaron los USD 845.58 millones en el trimestre analizado» (BCE, 2020). Por otro lado, de acuerdo a datos de la Asociación de Bancos Privados de Ecuador (AsOBANCA), hasta diciembre del 2019, el 53\% (USD 5198 millones) del total de colocación de la cartera de las cooperativas grandes, que atiende a sectores de menores ingresos y de la economía popular y solidaria, se dirigió a créditos de consumo, con un crecimiento en el del 12,3\% en el último año (ASOBANCA, 2020). Este mayor endeudamiento para consumo en un contexto de menor crecimiento económico ${ }^{26}$ puede convertirse en un riesgo para la economía y las familias.

En un contexto de mayor dependencia de los hogares hacia los ingresos obtenidos en el mercado de trabajo y el crédito privado - consumo, tarjetas de crédito, etc.—, existen mayores posibilidades de que los efectos de las reformas acordadas con el FMI en las economías domésticas sean paliados a través del mayor endeudamiento individual y familiar, obligando a que los costos sociales y económicos de la crisis, los recortes y la privatización sean asumidos por los sectores de la sociedad más vulnerables.

De acuerdo a Gago y Cavallero (2019), en su lectura feminista sobre los efectos de la deuda y el ajuste estructural en Argentina:

La deuda es lo que suple infraestructuras básicas de la vida: servicios de salud que no se tienen, insumos ante la llegada de unx hijx, la compra de una moto para poder trabajar de delivery. La deuda es el recurso que aparece ante las emergencias frente al despojo de otras redes 
de apoyo [...]. La deuda flexibiliza compulsivamente las condiciones de trabajo que deben aceptarse, y en ese sentido es un dispositivo eficaz de explotación. La deuda, entonces, organiza una economía de la obediencia que es, ni más ni menos, que una economía específica de la violencia. (2019, pág. 15)

De esta manera, el endeudamiento de las economías domésticas para enfrentar la crisis, implica que los trabajos vinculados a la reproducción y el cuidado se conviertan, gracias a su vínculo con las finanzas, en espacios que facilitan la acumulación del capital financiero (Gago y Cavallero, 2019) o como sostiene Harvey (2005) que sean parte de los procesos de acumulación por desposesión que operan a través de la deuda.

\section{SUMISAS}

Como se ha visto a lo largo de este artículo, los diálogos entre feminismo y marxismo muestran que el lugar que ocupan las mujeres en el capitalismo ha significado el desarrollo permanente de mecanismos para que cumplan su trabajo en la reproducción de la vida y el sostenimiento del cuidado. Estos se basan, además, en cumplir el destino manifiesto de la maternidad: el capitalismo «siempre ha necesitado controlar el cuerpo de las mujeres porque es un sistema de explotación que privilegia el trabajo como fuente de su riqueza de acumulación» (Federici, 2014). De esta manera, el capitalismo construye la idea y el orden social donde «el cuerpo de la mujer empieza a ser visto como una máquina para la producción de fuerza de trabajo. El útero es mirado literalmente como una fábrica de trabajadores» (Federici, 2014). Este modo de producción ha desplegado mecanismos que someten al lugar reproductivo e instaura un mecanismo de disciplinamiento sobre la vida de las mujeres mediante el control del Estado, el capital y el poder patriarcal.

En la actualidad, el capitalismo se encuentra en una fase del capital muy similar a la acumulación originaria, es decir, a un momento profundamente violento. El aumento agresivo de la violencia contra las mujeres y el ejercicio de la crueldad, como quiebre del proyecto empático entre el ser humano y la vida, son muestras de esta fase porque develan que el proyecto histórico, tanto del capital como del patriarcado, es su sometimiento al rol de reproductoras y cuidadoras. En ese sentido, todo intento por no cumplir con ese mandato obligatorio de la reproducción y tomar decisiones sobre nuestras vidas y cuerpos, se paga con violencia (Segato, 2016).

Bajo esa lupa, el aumento de feminicidios en Ecuador en los últimos años es sin duda un elemento central en el carácter y la manera en cómo se despliegan capitalismo y patriarcado. Desde el año 2014, hay más de 642 feminicidios perpetrados en entornos cercanos, lo cual nos arroja la escalofriante cifra de que cada tres días es asesinada una mujer en el Ecuador (El Comercio, 2019a).

No es nuestro propósito de este estudio entrar en detalles sobre cuáles fueron las políticas públicas que se desplegaron en esta última década, lo cierto es que a pesar de contar desde el 2018 con una Ley de Prevención y Erradicación de las Violencias de Género, el Gobierno ecuatoriano redujo el presupuesto para la implementación de la prevención de la violencia contra las mujeres de más de USD 5 millones (2019) a USD 876.000 (2020). Asimismo, a inicios del 2020, la mitad del personal del Servicio de Protección Integral para víctimas de violencia (SPI) fue despedido. ${ }^{27} \mathrm{El}$ argumento que el Estado formuló para semejante decisión fue que estaban optimizando recursos; mientras tanto, la violencia contra las mujeres permanece, se refuerza y queda en la total impunidad. 
En ese sentido, el supuesto compromiso del Estado con la vida y la vida sin violencia de las mujeres que consta en el texto de la Carta de Intención (LOI-MEFP, 2019): «Estamos en compromiso con la reducción de la violencia de género en todas sus manifestaciones, como demuestra legislación recientemente aprobada para prevenirla y erradicarla» (pág. 10), es una falacia. La falta de presupuesto y de voluntad del Estado para prevenir y erradicar la violencia de género es uno de los elementos centrales para la fosilización de una ley que no tienen forma de ser aplicada.

A esta indefensión se suma la producción de frustración de la población masculina que, con la concreción de las medidas de ajuste de la Carta de Intención, aumentará con la situación de crisis y angustia que el endeudamiento agresivo de las familias, los despidos y la precarización traerán. Como los estudios sobre empobrecimiento sostienen, existe una relación directa entre violencia económica y de género, a mayor violencia laboral y falta de dinero, los hombres son mucho más propensos a descargar esa violencia que reciben en sus familias (Voglio, 2012).

Por otro lado, el acuerdo con el FMI conlleva a los recortes y el desfinanciamiento en el sistema de salud pública y la seguridad social, y de esta manera impide que se garanticen los derechos sexuales y reproductivos, las maternidades plenas y la interrupción del embarazo, fundamentalmente para las mujeres empobrecidas y las más jóvenes. Es así que el Gobierno eliminó el presupuesto para los planes de prevención de embarazos adolescentes y recortó el presupuesto para salud en USD 81,1 millones. Este recorte en el presupuesto de la inversión social configura la idea de que la seguridad y salud privadas son las únicas vías posibles de atención. El reforzamiento del modelo privado implica una elitización en el acceso y el aumento de privilegios de clase de quienes pueden pagar por salud y seguridad.

Como correlato a la ampliación de las brechas y desigualdades bajo un modelo de privatización, la posibilidad de garantizar de manera pública, gratuita y libre la interrupción de embarazos no deseados así como maternidades plenas, se restringirá para la mayoría de las mujeres. Un acuerdo con el FMI no solo endeuda y precariza a las mujeres, también busca confinarlas al lugar de la sumisión, explotación y violencia que implica la reproducción social del capitalismo.

\section{REFLEXIONES FINALES}

Durante el primer trimestre del 2019, el Ministro de Hacienda argentino, Nicolás Dujovne, denominó a las políticas de ajuste estructural impulsadas por el Gobierno de Mauricio Macri como un "pacto entre caballeros», es decir, un pacto de confianza entre empresarios y funcionarios del Estado que buscaba congelar sesenta productos de la canasta básica, y así consolidar lo que Verónica Gago y Luci Cavallero (2019) han denominado «un pacto patriarcal de resolución de la crisis».

Este pacto patriarcal que se encuentra en el caso argentino puede arrojar luz para analizar el acuerdo entre el Estado ecuatoriano y el FMI, y sus posibles impactos en la vida de las mujeres. Como ha sostenido la economía feminista desde hace varios años, existe un conflicto entre el capitalismo y los procesos de reproducción de la vida, y simultáneamente hay una crisis de esta última, es decir, una crisis de todo aquello que permite habilitar la vida digna porque se trata de un sistema que expolia y mercantiliza la vida en todo su conjunto (Pérez Orozco, 
2018). A la precarización y sobreexplotación, se suma un elemento distinto a otros momentos del neoliberalismo: el endeudamiento agresivo en el ámbito privado que repercute directamente en las posibilidades de gestionar autónomamente la sobrevivencia y reproducción social, que está mayoritariamente en el trabajo de las mujeres. El ajuste, entonces, no solo tiene una dimensión pública, sino también cotidiana y privada, porque es en esta dimensión que se concreta el ajuste, cuando es la vida la que se ajusta.

Por otra parte, asumir esta relación intrínseca entre el capitalismo y el patriarcado, permite reflexionar sobre cómo las políticas neoliberales condicionan la posibilidad de decisión, justicia social y liberación de las mujeres, porque implica un «neoliberalismo disciplinador [que] es una forma concreta de poder estructural y conductual que combina el poder estructural del capital con formas más difusas y capilares de lo que M. Foucault llamó el poder disciplinador» (Gill y Roberts, 2011, pág. 162).

\section{NOTAS}

1 Se hace alusión a la afirmación There is no such thing as society. There are individual men and women, and there are families, realizada por Margaret Thatcher en una entrevista para la revista Women's Own, 890 , el 31 de octubre de 1987.

2 Con títulos como «Las mujeres más exitosas del mundo comparten sus mejores consejos de carrera» o «El momento de la "mujer de Davos"», 7 libros de mujeres poderosas que debes leer».

3 Como Patricia Botín, directora del Banco Santander; Meg Whitman, CEo de eBay; Randi Zuckerberg, CEO de Facebook; Cathy Engelbert, CEo de Deloitte; Mary Barra, presidenta de General Motors; Ginni Rometty, presidenta de IBM; Amy Hood, CEO de Microsoft.

4 La misma Christine Lagarde sostenía en su visita a Paraguay en el 2018: «Tienen que reducir las desigualdades. Las inequidades excesivas no permiten un crecimiento sostenido. Todos nuestros estudios avalan esa idea. Y la inclusión de las mujeres mejora el crecimiento. No es solo una obligación moral; también económica» (Lagarde, 2018).

5 En ese sentido, una de esas críticas frontales fue planteada por el Foro Feminista contra el G2o conformado en Argentina el año 2018, que reúne a distintos colectivos, organizaciones e investigadoras de América Latina.

6 Este artículo establece que «el Fondo supervisará el sistema monetario internacional a fin de asegurar su buen funcionamiento, y vigilará el cumplimiento por cada país miembro de sus obligaciones» (FMI, 2019). 7 Estos fueron los bonos: es decir, los préstamos en dólares emitidos por el Estado ecuatoriano en 2014, con fecha de vencimiento en 2024 a una rentabilidad del 11,5\% anual y una tasa de interés del 8,75\% nominal anual.

8 Reformas laborales de 2014, que fomentan el trabajo a tiempo parcial; enmiendas constitucionales de 2015, que atentan contra el derecho a la sindicalización en el sector público; reformas laborales de 2016, que permiten la reducción de la jornada laboral.

9 There is no alternative es una frase utilizada por Margaret Thatcher en sus discursos para señalar que no había ninguna otra alternativa a sus políticas neoliberales.

10 Las acciones previas son «medidas que un país conviene en adoptar antes de que el Directorio Ejecutivo del FMI apruebe el financiamiento o complete el examen. Estas medidas garantizan que se establezca la base necesaria para que el programa tenga éxito o vuelva a encauzarse si se desvía de las políticas acordadas» (FMI, 2016a).

11 Fueron despedidos 11.820 trabajadores del sector público desde diciembre 2018 según el propio Ministro de Trabajo del régimen.

12 Desembolsables en los próximos tres años 
13 Los préstamos obtenidos en el marco del sAF están dirigidos a países con graves problemas de balanzas de pago debido a deficiencias estructurales que tardan tiempo en resolverse. A diferencia de un préstamo en el marco de un acuerdo Stand-by, su duración es de mediano plazo - de tres años, con probabilidad de prórroga por 1 año- y su período de reembolso es más largo —entre $4 \frac{1}{2} 2$ y 10 años-con pagos en doce cuotas semestrales iguales (FMI, 2016).

14 La condicionalidad específica «abarca tanto el diseño de los programas que respalda la institución -es decir, las políticas macroeconómicas y estructurales-como las herramientas específicas utilizadas para supervisar los avances hacia las metas descritas por el país con la ayuda del FMI» (FMI, 2016a).

15 El texto constitucional es contundente en cuanto a instrumentos comerciales internacionales. Estos no pueden perjudicar «directa o indirectamente, el derecho a la salud, el acceso a medicamentos, insumos, servicios, ni los avances científicos y tecnológicos» (Constitución, 2008, Art. 186).

16 Entre otros: la falta de transparencia de todo el proceso así como del contenido de los textos - que no pasaron por la aprobación previa del ente legislativo- $y$, en esa medida, se obstaculiza el debate en la sociedad ecuatoriana y se incumple el derecho a la información y a la consulta

17 Es el tiempo destinado a las actividades domésticas, de cuidado y de apoyo a la comunidad sin obtener un pago o remuneración. Las horas de TNR corresponden al tiempo empleado por hombres y mujeres en el mantenimiento del hogar, actividades culinarias, cuidados de salud, etc. La carga de trabajo doméstico recae principalmente en mujeres jóvenes; mayoritariamente entre los 18 a 29 años —edad crucial para culminar los estudios superiores - y entre los 30 y 44 años —etapa activa en el mercado laboral - (INEC, 2019).

18 Es decir, que por cada USD 100 de gasto en consumo, los hogares ahorran USD 32

19 Medida a través del VAB

20 «La relación entre estas actividades y el trabajo se ha considerado también un indicador compuesto de bienestar (o su carencia)» (CNIG, 2016, pág. 33)

21 Datos de crecimiento economía ecuatoriana.

22 En 2018 el 46,5\% de los hombres y el 32,5\% de las mujeres tenían empleo pleno o adecuado, estas cifras disminuyen en el 2019 al 44,8\% y 30,6\%, respectivamente.

23 2018: hombres 5,1\%, mujeres 16,8\%; 2019: hombres 5,5\%, mujeres $18,4 \%$.

24 El subempleo de las mujeres aumentó de $15,5 \%$ al 16,2\% entre diciembre de 2018 y diciembre de 2019 . 25 «Esta carga es mayor para las mujeres en pobreza, por una serie de razones, entre ellas: composición familiar grande, menor acceso a servicios de trabajo remunerado, escasez de equipamiento de hogar para cuidados, menor acceso a servicios básicos — agua, por ejemplo-, falta de oportunidades de generación de ingresos propios, entre otros» (CNIG, 2016, pág. 67)

$26 \mathrm{El}$ producto interno bruto (PIB) decreció -0,1\% en el tercer trimestre de 2019 en comparación con similar período de 2018 (ВСЕ, 2020).

2760 funcionarios, de 120 que laboraban en el SPI, recibieron la notificación sobre que no se les renovará sus contratos, con el argumento de que es en cumplimiento del plan de reducción del Estado, dispuesto por el presidente Lenín Moreno.

\section{REFERENCIAS}

Acosta, A. (2014, septiembre 14). Otra vez bailando con lobos: el retorno del Ecuador al redil del FMI. Plan v. Recuperado de https://www.planv.com.ec/historias/sociedad/otra-vez-bailando -con-lobos-el-retorno-del-ecuador-al-redil-del-fmi

Arruzza, C. (2016, marzo 8). Reflexiones degeneradas: patriarcado y capitalismo. Marxismo Crítico. Recuperado de https://marxismocritico.com/2016/03/o8/reflexiones-degeneradas-patri arcado-y-capitalismo/

Asamblea Nacional Constituyente. (2008). Constitución Política del Ecuador. Manabí, Ecuador: Asamblea Nacional. 
Asobanca. (2020, enero 23). Evolución de las Cooperativas. Diciembre 2019. Asobanca. Recuperado de http://www.asobanca.org.ec/publicaciones/evoluci\% $\mathrm{C}_{3} \% \mathrm{~B} 3 \mathrm{n}$-de-las-cooperativas/ evoluci\% $\mathrm{C}_{3} \% \mathrm{~B}_{3}$-de-las-cooperativas-diciembre-2019

Baéz, J. (2020, febrero 2). El retroceso en los indicadores laborales del Plan Nacional de Desarrollo 20172021, incluyendo el trabajo infantil. Unidad y estudios de Coyuntura. Recuperado de https://coyunturaisip.wordpress.com/2020/o2/o2/el-retroceso-en-los-indicadores-laborales-del-plan-nacional-de-desarrollo-2017-2021-incluyendo-el-trabajo-infantil/?fbclid=IWAR2qMcsorbhIx2bL2 XGMSAgGRBMrhDQIdxhchtm9_qnoDoHwhxB9_futzno

Banco Central del Ecuador (BCE). (2020, enero 2). La economía ecuatoriana decreció -0,1\% en el tercer trimestre de 2019. BCE. Recuperado de https://www.bce.fin.ec/index.php/boletines-de-prensaarchivo/item/1339-la-econom\% $\mathrm{C}_{3} \% \mathrm{ADa}$-ecuatoriana-decreci\% $\mathrm{C}_{3} \% \mathrm{~B}_{3}$-o1-en-el-tercer-trimestre-de-2019

Cañamero, D. (2019). Prólogo. En A. Tarin, y J. Rivas, La clase trabajadora, ¿sujeto de cambios en el siglo Xxi? Madrid, España: Siglo Xxi.

Castillo, J. G. y Salas, C. (2018). Estabilidad laboral y desigualdad del ingreso: una perspectiva de género. Cuestiones Económicas, (28), 149-18o. Recuperado de https://www.bce.fin.ec/cuestiones_economicas/images/PDFs/2018/especial/RCEEDICIONESPECIAL2018.pdf

Cavallero, L. y Gago, V. (2019, abril 25) La deuda es un pacto entre caballeros. Crisis. Recuperado de https:/latinta.com.ar/2019/04/la-deuda-es-un-pacto-entre-caballeros/

Consejo Nacional para la Igualdad de Género (CNIG). (2016). Economía del cuidado, trabajo remunerado y no remunerado. Quito, Ecuador: Sector Público Gubernamental.

Correa, R. (2007). Más que liberar mercados hay que liberar al país. Discurso del presidente de Ecuador. Quito, Ecuador: Gobierno del Ecuador.

Correa, R. (2013, abril 16). Caminos para salir de la crisis. El ser humano por sobre el capital. El caso ecuatoriano. Presidencia de la República de Ecuador. Recuperado de https://www.presidencia. gob.ec/wp-content/uploads/downloads/2014/o1/2013-04-16-CONFERENCIA-UnivTecBerlin.doc

Crédito Real. (2018). Por qué aumentar el número de mujeres en puestos de liderazgo empresarial. Crédito Real. Recuperado de https://www.creditoreal.com.mx/blog-credito/ por-que-aumentar-el-numero-de-mujeres-en-puestos-de-liderazgo-empresarial

Davis, A. (2004). Mujer, raza y clase. Madrid, España: Akal.

Development Alternatives with Women for a New Era (DAWN). (2018). Segundo webinario formativo «Feministas hacia el G2o». DAWN. Recuperado de http:/dawnnet.org/audio/ audio-completo-del-2do-webinario-formativo-feministas-hacia-el-g2o/

El Comercio. (13 de marzo de 2019). Gobierno: 11820 funcionarios públicos fueron despedidos desde diciembre. El Comercio. Recuperado de https://www.elcomercio.com/actualidad/trabajadoresdesvinculados-sector-publico-ecuador.html

El Universo. (28 de julio de 2017). La situación es crítica, asegura Lenín Moreno al presentar el diagnóstico de la economía de Ecuador. El Universo. Recuperado de https://www.eluniverso.com/noticias/2017/07/28/nota/6302644/situacion-es-critica-asegura-lenin -moreno-presentar-diagnostico

EMOL. (2014, abril 13). Christine Lagarde: El FMI posee nuevo rostro y ya no impone ajustes estructurales. EMOL. Recuperado de https://www.emol.com/noticias/economia/2014/04/13/655084/christine-lagarde-el-fmi-posee-nuevo-rostro-y-ya-no-impone-ajustes-estructurales.html 
Federici, S. (19 de mayo de 2014). Entrevista a Silvia Federici. Coordinadora Feminista y Federación Estatal de Organizaciones Feminista. Recuperado de http://www.feministas.org/silvia-federici-el-cuerpode-la.html

Federici, S. (2010). Calibán y la bruja. Mujeres, cuerpo y acumulación primitiva. Madrid, España: Traficantes de Sueños.

Federici, S. (2011, marzo 23). Las mujeres y las luchas contra la mercantilización de la universidad (Entrevista). Sin Permiso. Recuperado de http://www.sinpermiso.info/textos/ las-mujeres-y-las-luchas-contra-la-mercantilizacin-de-la-universidad-entrevista

Federici, S. (2013). El feminismo y las políticas de lo común en una era de acumulación primitiva. New York, USA: Revolution at Point Zero.

Fondo Monetario Internacional (FMI). (2011). Convenio constitutivo del Fondo Monetario Internacional. IMF. Recuperado de https://www.imf.org/external/spanish/pubs/ft/aa/aa.pdf

Fondo Monetario Internacional (FMI). (2016). Ficha Técnica La condicionalidad del FMI. IMF. Recuperado de https://www.imf.org/external/np/exr/facts/spa/conditios.htm

Fondo Monetario Internacional (FMI). (2016). Ficha Técnica Servicio Ampliado del FMI (SAF). IMF. Recuperado de https://www.imf.org/external/np/exr/facts/spa/effs.htm

Fondo Monetario Internacional (FMI). (2018, julio 5). Comunicado de Prensa n. ${ }^{\circ}$ 18/278. IMF. Recuperado de https://www.imf.org/es/News/Articles/2018/07/05/pr18278-imf-staff-completes-2018-article -iv-mission-to-ecuador

Fondo Monetario Internacional (FMI). (2019, febrero 21). Comunicado de Prensa n. ${ }^{\circ}$ 19/52. IMF. Recuperado de https://www.imf.org/es/News/Articles/2019/02/21/pr1952-ecuador-and-imf -reach-staff-level-agreement-on-extended-fund-facility

Gill, S. and Adrienne, R. (2011). Macroeconomic governance, gendered inequality, and global crises. En I. B. Brigitte Young, Questioning financial governance from a feminist perspective (pp. 171-198). New York, USA: Routledge.

Harvey, D. (2005). El «nuevo» imperialismo: acumulación por desposesión. Buenos Aires, Argentina: CLACso. Harvey, D. (2014). Breve historia del neoliberalismo. Madrid, España: Ediciones Akal s. A.

Himmelweit, S. (2018). Feminist economics. Why all economists should be feminist economists. En An introduction to pluralist economics. Rethinking Economics. New York, UsA: Roudedge.

Hochschild, A. R. (1995). The culture of politics: traditional, post-modern, coldmodern, and warm-modern ideals of care. Social Politics, 2(3), 331-346. doi:https://doi.org/10.1093/sp/2.3.331

Instituto Ecuatoriano de Estadística y Censos (INEC). (2018). Encuesta Nacional de Empleo, Subempleo y Desempleo. InEc. Recuperado de http://www.ecuadorencifras.gob.ec/documentos/web-inec/ EMPLEO/2018/Diciembre-2018/Boletin\%2otecnico\%2ode\%2oempleo\%2odiciembre\%202018.pdf

Instituto Ecuatoriano de Estadística y Censos (INEC). (2019). Aporte del Trabajo No Remunerado de la Mujer a la Economía Nacional. Presentación Ix Semana Internacional de la Economía-PUCE. Quito, Ecuador.

Instituto Ecuatoriano de Estadística y Censos (INEC). (2020, enero 16). Boletín Técnico Encuesta Nacional de Empleo, Desempleo y Subempleo (ENEMDu). INEc. Recuperado de https://www.ecuadorencifras.gob.ec/documentos/web-inec/EMPLEO/2019/Diciembre/Boletin_tecnico_de_empleo_dic19.pdf

La Jornada. (2009, agosto 10). Asume Rafael Correa su segundo mandato como presidente de Ecuador. La Jornada. Recuperado de https://www.jornada.com.mx/2009/o8/11/mundo/o19nımun\# 
Lagarde, C. (2016, noviembre 18). El argumento económico en favor de empoderar mujeres. IMF. Recuperado https://www.imf.org/es/News/Articles/2016/11/18/sP111816-The-Business-Case -for-Womens-Empowerment

Lagarde, C. y Ostry, J. D. (2018, noviembre 29). Las ventajas económicas de la inclusión de género: Aún mayores de lo que se pensaba. Diálogo a fondo. Recuperado de https://blog-dialogoafondo.imf. org $/ ? \mathrm{p}=10372$

Lagarde, Ch. and Solberg, E. (2018, January 20). Why 2018 must be the year for women to thrive? World Economic Forum. Recuperado de https://www.weforum.org/agenda/2018/o1/the-time-has -come-for-women-to-thrive-heres-how/

Lawson, M., Chan, M.-K., Rhodes, F., Parvez Butt, A., Marriott, A., Ehmke, E., Jacobs, D., Seghers, J., Atienza, J. y Gowland R. (2019, enero 21). ¿Bienestar público o beneficio privado? oxfaM. Recuperado de https://www.oxfam.org/es/informes/bienestar-publico-o-beneficio-privado

Lawson, M., Parvez Butt, A., Harvey, R., Sarosi, D., Coffey, C., Piaget, K. y Thekkudan, J. (2020, enero 20). Tiempo para el cuidado. oxfam. Recuperado de https://www.oxfam.org/es/informes/ tiempo-para-el-cuidado

Líderes. (2016, marzo 16). Mujeres cabeza de familia son las más ahorradoras de Latinoamérica. Revista Líderes. Recuperado de https://www.revistalideres.ec/lideres/mujeres-cabeza-familia-ahorradoras-economia.html

Líderes. (2018, marzo 8). Se necesitan más mujeres en puestos de liderazgo. Revista Líderes. Recuperado de https:/lideresmexicanos.com/noticias/mujeres-en-puestos-de-liderazgo-ventaja-competitiva/.

LinkedIn Economic Graph (2017). The Gender Gap: To close gender gaps in the future, we need more women in emerging jobs today. LinkedIn Economic Graph. Recuperado de https://economicgraph.linkedin.com/blog/the-gendergap-to-close-gender-gaps-in-the-future-we-need-morewomen-in-emerging-jobs-tod

LOI-MefP. (2019, marzo 1). Carta de Intención de Acuerdo con el FMI. Recuperado de https://www.finanzas.gob.ec/wp-content/uploads/downloads/2019/o3/LOI-MEFP-Espa\%C3\%B1ol.pdf

Marcal, K. (2018). ¿Quién le hacía la cena a Adam Smith? Una historia de las mujeres y la economía. Barcelona, España: Editorial Debate.

Martínez, E. (2011, agosto 9). Capitalismo y patriarcado: la doble desigualdad de la mujer. Pueblos. Revista de Información y Debate. Recuperado de http://www.revistapueblos.org/old/spip.php?article2227

oxfam. (2017). Ocho personas poseen la misma riqueza que la mitad más pobre de la humanidad. oxfAM. Recuperado de https://www.oxfam.org/es/notas-prensa/ocho-personas-poseen-la-misma -riqueza-que-la-mitad-mas-pobre-de-la-humanidad

Pérez Orozco, A. (2006). Amenaza Tormenta: La crisis de los cuidados y la reorganización del sistema económico. Revista de Economía Crítica, (5), 7-37.

Rodríguez, C. (2015). Economía feminista y economía de cuidado. Aportes conceptuales para el estudio de la desigualdad. Nueva Sociedad, (256). Recuperado de http://nuso.org/media/articles/downloads/4102_1.pdf

Schild, V. (2016). Feminismo y neoliberalismo en América Latina. Nueva Sociedad, (265). Recuperado de https://nuso.org/articulo/feminismo-y-neoliberalismo-en-america-latina/

Secretaria Nacional de Planificación y Desarrollo (senplades). (2009). Plan Nacional para el Buen Vivir 2009-2013: Construyendo un Estado Plurinacional e Intercultural. Quito, Ecuador: SENPLADES. 
Segato, R. (2016). La guerra contra las mujeres. Recuperado de https://www.traficantes.net/sites/default/ files/pdfs/map45_segato_web.pdf

Segato, R. (2018). Conferencia magistral. Iv Cumbre Iberoamericana de Agendas Locales de Género. Cuenca, Ecuador.

Segato, R. (2019, junio 10). 14 voces para comprender la crisis del capitalismo. La Línea de Fuego. Recuperado de https://lalineadefuego.info/2019/05/o1/rita-segato-el-capital-recrudece-las -practicas-patriarcales/

Thatcher, M. (1981, mayo 3). Interview for Sunday Times. Margaret Thatcher Foundation. Recuperado de https://www.margaretthatcher.org/document/104475

Volio Monge, R. (2012). Reflexiones necesarias en torno al binomio violencia y pobreza de las mujeres. Revista Europea de Derechos Fundamentales, (19), 95-122. Recuperado de https://dialnet.unirioja. es/servlet/articulo? codigo $=4055498$

World Economic Forum. (2018). The Global Gender Gap Report 2018. World Economic Forum. Recuperado de https://www.weforum.org/reports/the-global-gender-gap-report-2018

Zafra, R. (2017). El entusiasmo. Precariedad y trabajo creativo en la era digital. Barcelona, España: Anagrama. 